\title{
Extension of the Arc Spectra of Palladium and Platinum (6500 to $12000 \mathrm{~A}$ )
}

\author{
Karl G. Kessler, William F. Meggers, and Charlotte E. Moore
}

\begin{abstract}
The arc spectra of palladium and platinum have been reinvestigated photographically in the red and infrared, and extended approximately 2000 angstroms beyond the former limit of observation. The spectra were excited in conventional direct-current ares between pure-metal electrodes and recorded by modern sensitized emulsions with the aid of diffraction gratings of $640-\mathrm{cm}$ radius. Improved wavelength values and intensity estimates are given for $63 \mathrm{Pd}$ I lines between 6508.42 and $11556.27 \mathrm{~A}$, and for $74 \mathrm{Pt}$ I lines batween 6643.32 and 10757.78 A. All of the observed $\mathrm{Pd}$ I lines have been explained as transitions between known atomic energy levels, but only 42 of the $74 \mathrm{Pt}$ I lines can be explained in like manner. It is concluded that further progress in the structural analysis of the Pt I spectrum is dependent on making an improved description in the visible and ultraviolet.
\end{abstract}

\section{Introduction}

Inspiration for this investigation was provided by the current compilation of NBS Circular 467 on atomic energy levels [1]. ${ }^{1}$ Such analyses are usually handicapped by the limited range of photographic observation of spectra, especially in the inirared. Thirty years ago one of the present authors [2] recorded some inirared lines in the arc spectra of the platinum elements by sensitizing ordinary photographic emulsions with dicyanin. Although considerable extensions of the arc spectra were thus made, the greatest wavelength then detected photographically with palladium was $9234.02 \mathrm{~A}$, and with platinum, $8762.48 \mathrm{~A}$.

In 1935 new types of photographic emulsions and sensitizing dyes for infrared photography were described [3], and good inirared sensitive photographic plates became commercially available for the first time. By using these plates the arc spectra of many elements were promptly observed to a new limit in the inirared several thousand angstroms beyond the previous limit set by dicyanin. Such observations for some noble metals were recently made for rhodium [4] and ruthenium [5], and are here presented for palladium and platinum. In most cases, as in rhodium, extension of the inirared spectrum has been rewarded by the discovery of new energy levels, and the possibility that this might happen also in palladium and platinum could not be neglected. The present paper has three collaborators: the first made the spectrograms, the second measured the wavelengths, and the third interpreted the spectra.

\section{Materials and Methods}

Solid, cylindrical electrodes, approximately $6 \mathrm{~mm}$ in diameter and $8 \mathrm{~mm}$ long; were made by pressing the purest powdered palladium or platinum metal in a Dietert hydraulic press. These electrodes were pinched in slotted copper rods that served as electrode holders. Electric arcs were produced between

Figures in brackets indicate the literature references at the end of this paper. a pair of electrodes by currents of 6 to 7 amp irom a 220-v d-c circuit. In the case of platinum, the arc was extremely unstable and could not be maintained in the slotted copper holders, but the stability was satisiactory when the short platinum electrodes were beld by water-cooled brass clamps.

To record the red and near infrared spectra of these metal ares, three types of sensitized photographic plates were used. The wavelength range 6500 to 8800 A was photographed with Eastman I-N plates, 8500 to 10500 A with Eastman I-Q plates, and 10000 to $12000 \mathrm{~A}$ with Eastman I-Z plates. Before use, these plates were hypersensitized by bathing in dilute solutions of ammonia, followed by rinsing in alcohol and rapid drying with an air blower.

The previous inirared spectrograms obtained with dicyanin-stained plates [2] were made with a concave grating, of $640-\mathrm{cm}$ radius, ruled with 7,500 lines per inch; the scale of the spectrum was $10.4 \mathrm{~A} / \mathrm{mm}$. Because the commercial inirared-sensitive photographic plates are vastly more sensitive, it was possible to make the new spectrograms with increased dispersion. Accordingly, the range recorded on I-N plates was observed with a grating ruled 30,000 lines per inch and an average reciprocal dispersion of $1.6 \mathrm{~A} / \mathrm{mm}$. The ranges covered by I-Q and I-Z plates were photographed with a grating ruled 15,000 lines per inch and reciprocal dispersion of $4.5 \mathrm{~A} / \mathrm{mm}$. Both gratings have radii of $640 \mathrm{~cm}$ and are illuminated with parallel light in Wadsworth mountings.

The Eastman I-N plates were so extraordinarily sensitive that arc spectra were completely recorded with continuous background in $30 \mathrm{~min}$. Relatively, the I-Q and I-Z plates are much less sensitive, so that exposure times of 2 to $4 \mathrm{hr}$. were required to record spectra with background. Furthermore, the sensitivity of I-Z plates declines rapidly beyond $11000 \mathrm{~A}$, the background vanishes even with 4-hr exposures, and the estimated relative intensities of observed spectral lines are consequently too low for the longest waves.

The red and infrared arc spectra ofi palladium and 
platinum were photographed in the first order of both gratings, and for the measurement of wavelengths the arc spectrum of iron was recorded in juxtaposition. In the I-N region the wavelengths were usually measured relative to the first-order spectrum of iron, but in the I-Q and I-Z ranges the standards were generally taken from the second-and third-order iron spectra, respectively. The spectrograms were measured on a 20-inch Gaertner comparator reading directly to 1 micron.

\section{Results}

The experimental results of this investigation consist of measured wavelengths and estimated relative intensities of palladium and platinum lines recorded photographically in the red and infrared. The wavelengths result from averaging 2 to 4 independent determinations; their average uncertainty is of the order of $\pm 0.01 \mathrm{~A}$. Estimated intensities are based largely on the strongest spectrogram; they are given on an open scale ranging from 1 to 2000 , to represent approximate relative energies (except beyond $11000 \mathrm{~A}$, where sensitivity of detection falls off rather rapidly). Some of the intensity numbers are accompanied by letters that have been recommended by the Joint Commission for Spectroscopy [6] for the qualitative description of spectral lines. Briefly, $h$ stands for hazy, $H$ for very hazy, $l$ for shaded to longer waves, and $s$ for shaded to shorter waves.

In the range of wavelengths reported here, the only spectral impurities detected in palladium and platinum were potassium and calcium. However, the arc spectra of noble metals, especially in the long-wave range, are always contaminated by superposed spectra of atmospheric constituents and metallic compounds. In addition, the arc spectrum of platinum in air is accompanied by fairly complete atomic spectra of nitrogen, oxygen, and a strong background of irregularly spaced lines that represent certain unidentified molecules. That background is usually present in all arc spectra of noble metals, and can therefore be recognized and ignored by recording side by side the arc spectra of two different noble metals and by measuring only the lines that are not common to both.

\subsection{Palladium (46 Pd)}

Among the six noble metals, palladium is outstanding in possessing the simplest arc spectrum. Following previous workers, Shenstone [7] in 1930 compiled and thoroughly analyzed the arc spectrum of palladium. That compilation lists about 350 Pd I lines with wavelengths ranging from 1945.98 to $9234.02 \mathrm{~A}$. Most of those lines were quoted from published [2] and unpublished data by Meggers. Recently, Shenstone [8] made a new examination of the $\mathrm{Pd}$ I spectrum and contributed 75 new lines, mostly in the ultraviolet down to $1683.65 \mathrm{~A}$.

In the present paper only new data for 63 red and infrared lines of the $\mathrm{Pd}$ I spectrum are given in table 1. Between 6508 and $8761 \mathrm{~A}$ the new list is almost identical with that published by Meggers [2] in 1925, except that the intensity scale has been increased, and the accuracy of wavelengths has been improved. A few faint lines in the early list were not confirmed, but 17 new lines were observed, and the Pd I spectrum has been extended to $11556.27 \mathrm{~A}$ in the infrared. All the new lines were immediately explained as transitions between atomic energy levels already published by Shenstone [8], and it must be concluded that the analysis of the $\mathrm{Pd}$ I spectrum is practically complete, so that further effort to extend it may not be justified. Incidentally, two forbidden transitions (6915 and $7147 \mathrm{~A}$ ) between terms of like parity, first discovered by Shenstone [8], were nicely confirmed by the present observations.

TABLE 1. Extension of the first spectrum of palladium, $\mathrm{Pd}$ I

[ $h$, hazy: $H$, very hazy: $l$, shaded to longer waves: $s$, shaded to shorter waves]

\begin{tabular}{|c|c|c|c|c|c|c|c|c|c|}
\hline \multirow{2}{*}{$\begin{array}{l}\text { Wave- } \\
\text { length }\end{array}$} & \multirow{2}{*}{$\begin{array}{c}\text { Intensity } \\
\text { and } \\
\text { char- } \\
\text { acter }\end{array}$} & \multicolumn{2}{|c|}{ Wave number, $K$} & \multirow{2}{*}{$\begin{array}{l}\text { Spectral-term } \\
\text { combination }\end{array}$} & \multirow{2}{*}{$\begin{array}{l}\text { Wave- } \\
\text { length }\end{array}$} & \multirow{2}{*}{$\begin{array}{c}\text { Intensity } \\
\text { and } \\
\text { char- } \\
\text { acter }\end{array}$} & \multicolumn{2}{|c|}{ Wave number, $K$} & \multirow{2}{*}{$\begin{array}{l}\text { Spectral-term } \\
\text { combination }\end{array}$} \\
\hline & & Observed & $\begin{array}{l}\text { Com- } \\
\text { puted }\end{array}$ & & & & Observed & $\begin{array}{l}\text { Com- } \\
\text { puted }\end{array}$ & \\
\hline$A$ & & $\mathrm{~cm}^{-1}$ & $\mathrm{~cm}^{-1}$ & & $A$ & & $\mathrm{~cm}^{-1}$ & $\mathrm{~cm}^{-1}$ & \\
\hline 6508.42 & 30 & 15360.47 & 60.5 & $5 p{ }^{3} \mathrm{D}_{2}^{\circ}-6 s{ }^{3} \mathrm{D}_{1}$ & 6774.53 & 200 & 14757. 10 & 57. 0 & $5 s^{2} \quad{ }^{3} \mathrm{~F}_{4}-5 p{ }^{1} \mathrm{~F}_{3}^{\circ}$ \\
\hline 6591.44 & 5 & 15167. 00 & $6 \% .1$ & $5 p{ }^{1} \mathrm{~F}_{3}^{\circ}-5 d^{3} \mathrm{~F}_{4}$ & 6784.51 & 1000 & 14735. 39 & 35.4 & $5 p{ }^{3} \mathrm{P}_{2}^{\circ}-6 s{ }^{3} \mathrm{D}_{3}$ \\
\hline 6597.06 & 1 & 15154. 08 & 54.1 & $5 p{ }^{1} \mathrm{~F}_{3}^{\circ}-5 d{ }^{3} \mathrm{~F}_{3}$ & 6833. 41 & $80 h$ & 14629.95 & 29.9 & $5 p{ }^{3} \mathrm{D}_{1}^{\circ}-5 d^{3} \mathrm{D}_{2}$ \\
\hline 6603.03 & 1 & 15140.38 & 40.4 & $5 p \quad{ }^{1} \mathrm{~F}_{3}^{\circ}-5 d d^{3} \mathrm{D}_{2}$ & 6878.36 & 3 & 14534. 34 & 34. 3 & $5 p{ }^{1} \mathrm{P}_{1}^{\circ}-5 d{ }^{3} \mathrm{P}_{0}$ \\
\hline 6623.27 & 20 & 15094. 11 & 94.2 & $5 p{ }^{3} \mathrm{D}_{3}^{\circ}-6 s{ }^{1} \mathrm{D}_{2}$ & 6915. 0 & $5 H s$ & 144457.3 & 57.1 & $5 p{ }^{3} \mathrm{D}_{1}^{\circ}-6 p{ }^{3} \mathrm{P}_{1}^{\circ}$ \\
\hline 6625. 28 & 10 & 15089.53 & 89.6 & $5 p \quad{ }^{1} \mathrm{~F}_{3}^{\circ}-5 d{ }^{3} \mathrm{D}_{3}$ & 6916.53 & $200 h l$ & 14454. 13 & 54.1 & $5 p{ }^{3} \mathrm{D}_{1}^{\circ}-5 d{ }^{3} \mathrm{P}_{1}$ \\
\hline 6662.87 & 20 & 15004.40 & 04.4 & $5 p^{3} \mathrm{D}_{1}^{\circ}-5 d{ }^{3} \mathrm{P}_{0}$ & 6917.53 & 5 & 14452. 04 & 52. 0 & $5 p{ }^{3} \mathrm{D}_{1}-5 d{ }^{3} \mathrm{P}_{2}$ \\
\hline 6681.56 & 5 & 14962. 43 & 62.5 & $5 p{ }^{1} \mathrm{~F}_{3}^{\circ}-5 d{ }^{3} \mathrm{P}_{2}$ & 7016.47 & 200 & 14248. 25 & 48. 3 & $5 p{ }^{3} \mathrm{P}_{0}^{\circ}-6 s{ }^{3} \mathrm{D}_{1}$ \\
\hline 6685.70 & 3 & 14953. 17 & 53. 0 & $5 p{ }^{1} \mathrm{~F}_{3}^{\circ}-5 d d^{3} \mathrm{G}_{4}$ & 7026.88 & 2 & 14227. 15 & 27. 2 & $5 p{ }^{1} \mathrm{D}_{2}^{\circ}-5 d{ }^{3} \mathrm{D}_{2}$ \\
\hline 6686.80 & 20 & 14950.71 & 50.7 & $5 p \quad{ }^{3} \mathrm{P}_{2}^{\circ}-6 s \quad{ }^{3} \mathrm{D}_{2}$ & 7037.58 & 10 & 14205.51 & 05.5 & $5 p{ }^{3} \mathrm{D}_{1}^{\circ}-5 d{ }^{3} \mathrm{~S}_{1}$ \\
\hline
\end{tabular}


TABLE 1. Extension of the first spectrum of palladium, Pd I-Continued

[h, hazy: $H$, very hazy: $l$, shaded to longer waves; $s$, shaded to shorter waves]

\begin{tabular}{|c|c|c|c|c|c|c|c|c|c|}
\hline \multirow{2}{*}{$\begin{array}{l}\text { Wave- } \\
\text { length }\end{array}$} & \multirow{2}{*}{$\begin{array}{c}\text { Intensity } \\
\text { and } \\
\text { char- } \\
\text { acter }\end{array}$} & \multicolumn{2}{|c|}{ Wave number, $K$} & \multirow{2}{*}{$\begin{array}{l}\text { Spectral-term } \\
\text { combination }\end{array}$} & \multirow{2}{*}{$\begin{array}{l}\text { Wave- } \\
\text { length }\end{array}$} & \multirow{2}{*}{$\begin{array}{c}\text { Intensity } \\
\text { and } \\
\text { char- } \\
\text { acter }\end{array}$} & \multicolumn{2}{|c|}{ Wave number, $K$} & \multirow{2}{*}{$\begin{array}{l}\text { Spectral-term } \\
\text { combination }\end{array}$} \\
\hline & & Observed & $\begin{array}{l}\text { Com- } \\
\text { puted }\end{array}$ & & & & Observed & $\begin{array}{l}\text { Com- } \\
\text { puted }\end{array}$ & \\
\hline $\begin{array}{l}\text { A } \\
7052.04 \\
7060.29 \\
7115.82 \\
7147.5 \\
7149.00\end{array}$ & $\begin{array}{c}3 \\
50 \\
7 \\
2 \mathrm{Hs} \\
50 h \mathrm{l}\end{array}$ & $\begin{array}{c}c m^{-1} \\
14176.38 \\
14159.82 \\
14049.32 \\
13987.0 \\
13984.12\end{array}$ & $\begin{array}{l}c m^{-1} \\
76.4 \\
59.8 \\
49.3 \\
87.0 \\
84.0\end{array}$ & $\begin{array}{lll}5 p & { }^{1} \mathrm{D}_{2}^{\circ}-5 d & { }^{3} \mathrm{D}_{3} \\
5 p & { }^{1} \mathrm{P}_{1}-5 d & { }^{3} \mathrm{D}_{2} \\
5 p & { }^{1} \mathrm{D}_{2}^{\circ}-5 d & { }^{3} \mathrm{P}_{2} \\
5 p & { }^{1} \mathrm{P}_{1}^{\circ}-6 p & { }^{3} \mathrm{P}_{1} \\
5 p & { }^{1} \mathrm{P}_{1}-5 d & { }^{3} \mathrm{P}_{1}\end{array}$ & $\begin{array}{c}\text { A } \\
\text { 8599. } 13 \\
8644.48 \\
8695.12 \\
8761.39 \\
8922.04\end{array}$ & $\begin{array}{r}300 \\
100 \\
60 \\
600 \\
6 h\end{array}$ & $\begin{array}{c}\mathrm{cm}^{-1} \\
11625.89 \\
11564.90 \\
11497.55 \\
11410.58 \\
11205.12\end{array}$ & $\begin{array}{l}c m^{-1} \\
26.0 \\
65.0 \\
97.6 \\
10.7 \\
04.9\end{array}$ & $\begin{array}{lll}5 p & { }^{3} \mathrm{D}_{3}^{\circ}-6 s & { }^{3} \mathrm{D}_{2} \\
5 p & { }^{1} \mathrm{D}_{2}^{\circ}-6 s & { }^{3} \mathrm{D}_{1} \\
5 p & { }^{1} \mathrm{P}_{1}-6 s & { }^{3} \mathrm{D}_{1} \\
5 p & { }^{3} \mathrm{D}_{3}^{\circ}-6 s & { }^{3} \mathrm{D}_{3} \\
6 s & { }^{3} \mathrm{D}_{2}- & 27_{2}^{\circ}\end{array}$ \\
\hline $\begin{array}{l}7150.13 \\
7242.91 \\
7278.44 \\
7310.08 \\
7368.12\end{array}$ & $\begin{array}{r}2 \\
5 \\
4 \\
200 \\
1000\end{array}$ & $\begin{array}{l}\text { 13981. } 91 \\
13802.80 \\
13735.42 \\
13675.97 \\
13568.24\end{array}$ & $\begin{array}{l}81.9 \\
02.8 \\
35.4 \\
76.0 \\
68.2\end{array}$ & $\begin{array}{lll}5 p & { }^{1} \mathrm{P}_{1}-5 d & { }^{3} \mathrm{P}_{2} \\
5 p & { }^{1} \mathrm{D}_{2}^{\circ}-5 d & { }^{3} \mathrm{~S}_{1} \\
5 p & { }^{1} \mathrm{P}_{1}^{\circ}-5 d & { }^{3} \mathrm{~S}_{1} \\
5 p & { }^{3} \mathrm{~F}_{2}^{\circ}-6 s & { }^{1} \mathrm{D}_{2} \\
5 p & { }^{3} \mathrm{~F}_{3}^{\circ}-6 s & { }^{3} \mathrm{D}_{2}\end{array}$ & $\begin{array}{l}8984.04 \\
9038.75 \\
9233.85 \\
9380.32 \\
9433.02\end{array}$ & $\begin{array}{r}40 \\
5 \\
1000 \\
200 \\
70\end{array}$ & $\begin{array}{l}\text { 11127. } 79 \\
11060.44 \\
10826.75 \\
10657.69 \\
10598.15\end{array}$ & $\begin{array}{l}27.7 \\
60.3 \\
26.7 \\
57.6 \\
98.2\end{array}$ & $\begin{array}{lll}5 s^{2} & { }^{3} \mathrm{~F}_{2}-5 p & { }^{1} \mathrm{~F}_{\mathrm{i}} \\
5 s^{2} & { }^{3} \mathrm{~F}_{2}-5 p & { }^{1} \mathrm{D}_{2}^{\circ} \\
5 s^{2} & { }^{3} \mathrm{~F}_{4}-5 p & { }^{3} \mathrm{~F}_{4}^{\circ} \\
5 s^{2} & { }^{3} \mathrm{~F}_{2}-5 p & { }^{3} \mathrm{D}_{1}^{\circ} \\
5 s^{2} & { }^{3} \mathrm{~F}_{3}-5 p & { }^{3} \mathrm{~F}_{2}^{\circ}\end{array}$ \\
\hline $\begin{array}{l}7391.92 \\
7486.93 \\
7763.99 \\
7786.63 \\
7915.79\end{array}$ & $\begin{array}{r}400 \\
300 \\
2000 \\
500 \\
800\end{array}$ & $\begin{array}{l}13524.56 \\
13352.93 \\
12876.43 \\
12838.99 \\
12629.50\end{array}$ & $\begin{array}{l}24.6 \\
52.9 \\
76.4 \\
39.0 \\
29.6\end{array}$ & $\begin{array}{lll}5 p & { }^{3} \mathrm{~F}_{2}^{\circ}-6 s & { }^{3} \mathrm{D}_{1} \\
5 p & { }^{3} \mathrm{~F}_{3}^{\circ}-6 s & { }^{3} \mathrm{D}_{3} \\
5 p & { }^{3} \mathrm{~F}_{4}^{\circ}-6 s & { }^{3} \mathrm{D}_{3} \\
5 p & { }^{3} \mathrm{P}_{1}^{\circ}-6 s & { }^{3} \mathrm{D}_{2} \\
5 p & { }^{1} \mathrm{~F}_{3}^{\circ}-6 s & { }^{1} \mathrm{D}_{2}\end{array}$ & $\begin{array}{r}9793.76 \\
9852.14 \\
10509.75 \\
10890.26 \\
10985.03\end{array}$ & $\begin{array}{c}150 \\
3 \\
7 h \\
80 \\
30\end{array}$ & $\begin{array}{r}10207.78 \\
10147.30 \\
9512.36 \\
9180.00 \\
9100.80\end{array}$ & $\begin{array}{l}07.8 \\
47.1 \\
12.4 \\
80.0 \\
00.7\end{array}$ & $\begin{array}{lll}5 p & { }^{3} \mathrm{~F}_{2}^{\circ}-6 s & { }^{3} \mathrm{D}_{2} \\
5 s^{2} & { }^{3} \mathrm{~F}_{2}-5 p & { }^{1} \mathrm{~F}_{3} \\
6 s & { }^{3} \mathrm{D}_{3}- & 24_{433}^{\circ} \\
5 s^{2} & { }^{3} \mathrm{~F}_{3}-5 p & { }^{3} \mathrm{D}_{3}^{\circ} \\
5 s^{2} & { }^{3} \mathrm{~F}_{2}-5 p & { }^{3} \mathrm{~F}_{2}^{\circ}\end{array}$ \\
\hline $\begin{array}{l}7961.03 \\
8132.81 \\
8249.19 \\
8300.82 \\
8353.58\end{array}$ & $\begin{array}{r}200 \\
400 \\
50 \\
1000 \\
200\end{array}$ & $\begin{array}{l}12557.73 \\
12292.49 \\
12119.07 \\
12043.69 \\
11967.63\end{array}$ & $\begin{array}{l}\text { 57. } 8 \\
92.4 \\
19.1 \\
43.7 \\
67.7\end{array}$ & $\begin{array}{lll}5 s^{2} & { }^{3} \mathrm{~F}_{3}-5 p & { }^{1} \mathrm{D}_{2}^{\circ} \\
5 s^{2} & { }^{3} \mathrm{~F}_{4}-5 p & { }^{3} \mathrm{D}_{3}^{\circ} \\
5 p & { }^{3} \mathrm{D}_{1}^{\circ}-6 s & { }^{1} \mathrm{D}_{2} \\
5 p & { }^{3} \mathrm{D}_{2}^{\circ}-6 s & { }^{3} \mathrm{D}_{2} \\
5 p & { }^{3} \mathrm{D}_{1}^{\circ}-6 s & { }^{3} \mathrm{D}^{1}\end{array}$ & $\begin{array}{l}11175.09 \\
11409.45 \\
11556.27\end{array}$ & $\begin{array}{r}10 \\
8 \\
5\end{array}$ & $\begin{array}{l}8946.02 \\
8762.26 \\
8650.94\end{array}$ & $\begin{array}{l}\text { 46. } 1 \\
62.3 \\
50.9\end{array}$ & $\begin{array}{lll}5 p & { }^{1} \mathrm{~F}_{3}^{\circ}-6 s & { }^{3} \mathrm{D}_{3} \\
5 s^{2} & { }^{3} \mathrm{~F}_{3}-5 p & { }^{3} \mathrm{D}_{2}^{\circ} \\
5 p & { }^{3} \mathrm{D}_{1}^{\circ}-6 s & { }^{3} \mathrm{D}_{2}\end{array}$ \\
\hline $\begin{array}{l}8387.99 \\
8451.93 \\
8532.76 \\
8582.10 \\
8585.33\end{array}$ & $\begin{array}{l}3 h \\
10 \\
300 \\
200 \\
20\end{array}$ & $\begin{array}{l}11918.53 \\
11828.37 \\
11716.32 \\
11648.96 \\
11644.58\end{array}$ & $\begin{array}{l}18.5 \\
28.4 \\
16.4 \\
49.0 \\
44.6\end{array}$ & $\begin{array}{lll}6 s & { }^{3} \mathrm{D}_{3}-5 p & { }^{1} \mathrm{G}_{4}^{\circ} \\
5 p & { }^{3} \mathrm{D}_{2}^{\circ}-6 s & { }^{3} \mathrm{D}_{3} \\
5 p & { }^{1} \mathrm{D}_{2}^{\circ}-6 s & { }^{1} \mathrm{D}_{2} \\
5 p & { }^{1} \mathrm{P}_{1}-6 s & { }^{1} \mathrm{D}_{2} \\
5 s^{2} & { }^{3} \mathrm{~F}_{3}-5 p & { }^{1} \mathrm{~F}_{3}^{\circ}\end{array}$ & & & & & \\
\hline
\end{tabular}

\subsection{Platinum (78 Pt)}

Although nearly 1,000 lines have been reported for the arc spectrum of platinum, the data must be sought in five different sources, because no one has made a systematic and satisfactory description of this spectrum. Haussman [9] in a report on Zeeman effect and terms in the $\mathrm{Pt}$ I spectrum compiled a list of 377 classified lines, quoting wavelengths from Meggers [2], Meggers and Laporte [10], Kayser [11], and Exner and Haschek [12]. The only additional data are wavelengths of 56 ultraviolet lines $(2241.20$ to $1928.85 \mathrm{~A}$ ) published by Livingood [13], but most of these remain unclassified.

The present paper is concerned primarily with improved description of the long-wave portion of the Pt I spectrum through the use of photographic plates of greater sensitivity and spectrographs of greater dispersive power. The pioneering work of Meggers [2] produced about $50 \mathrm{Pt}$ I lines with wavelengths greater than $6648 \mathrm{~A}$; now the number is increased to 74 as displayed in table 2 . The photographic extension is nearly $2000 \mathrm{~A}$, but the Pt I spectrum appears to be very sparce in the infrared, and no new lines could be detected beyond 10758 A with 4-hr exposures.

Only 42 of the Pt I lines in table 2 can be accounted for as combinations of Haussmann's [9] or Livingood's [10] terms. Attempts to classify the remainder by searching for new atomic energy levels among the unclassified lines were not successful. In fact, a critical examination of the old analyses of the Pt I spectrum raised many doubts about the interpretation of certain levels and the reality of others. A discussion of this will appear in Atomic Energy Levels [1]. It must be concluded that no further progress can be made with the structural analysis of the PtI spectrum without a more complete and homogeneous description of this spectrum. 
TABLE 2. Extension of the first spectrum of platinum, $\mathrm{Pt} \mathrm{I}^{\text {a }}$

$[h$, hazy $]$

\begin{tabular}{|c|c|c|c|c|c|c|c|c|c|}
\hline \multirow{2}{*}{$\begin{array}{l}\text { Wave- } \\
\text { length }\end{array}$} & \multirow{2}{*}{$\begin{array}{c}\text { Intensity } \\
\text { and } \\
\text { char- } \\
\text { acter }\end{array}$} & \multicolumn{2}{|c|}{ Wave number, $K$} & \multirow{2}{*}{$\begin{array}{l}\text { Spectral-term } \\
\text { combination }\end{array}$} & \multirow{2}{*}{$\begin{array}{l}\text { Wave- } \\
\text { length }\end{array}$} & \multirow{2}{*}{$\begin{array}{l}\text { Intensity } \\
\text { and } \\
\text { char- } \\
\text { acter }\end{array}$} & \multicolumn{2}{|c|}{ Wave number, $K$} & \multirow{2}{*}{$\begin{array}{l}\text { Spectral-term } \\
\text { combination }\end{array}$} \\
\hline & & Observed & $\begin{array}{l}\text { Com- } \\
\text { puted }\end{array}$ & & & & Observed & $\begin{array}{l}\text { Com- } \\
\text { puted }\end{array}$ & \\
\hline $\begin{array}{c}A \\
6648.32 \\
6655.55 \\
6710.41 \\
6760.01 \\
6820.21\end{array}$ & $\begin{array}{r}100 \\
3 \\
400 \\
1000 \\
10\end{array}$ & $\begin{array}{c}\mathrm{cm}^{-1} \\
15037.24 \\
15020.91 \\
14898.11 \\
14788.80 \\
14658.26\end{array}$ & $\begin{array}{l}\mathrm{cm}^{-1} \\
37.2 \\
20.9 \\
98.2 \\
88.6 \\
-\end{array}$ & $\begin{aligned} & 6_{2}^{\circ}-e^{3} \mathrm{D}_{3} \\
& 22_{3}^{\circ}-\mathrm{E}_{3} \\
& 8_{3}^{\circ}-e^{3} \mathrm{D}_{2} \\
& z^{3} \mathrm{~F}_{4}^{\circ}-e^{3} \mathrm{D}_{3}\end{aligned}$ & $\begin{array}{c}A \\
7786.77 \\
7790.21 \\
7830.42 \\
7877.45 \\
7911.26\end{array}$ & $\begin{array}{r}80 \\
5 h \\
6 h \\
3 h \\
20 h\end{array}$ & $\begin{array}{c}\mathrm{cm}^{-1} \\
\text { 12838. } 76 \\
\text { 12833. } 09 \\
\text { 12767. } 19 \\
12690.97 \\
12636.73\end{array}$ & $\begin{array}{c}\mathrm{cm}^{-1} \\
38.7 \\
-- \\
-- \\
-- \\
--\end{array}$ & 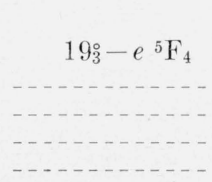 \\
\hline $\begin{array}{l}6838.08 \\
6842.61 \\
6896.73 \\
6908.82 \\
6956.85\end{array}$ & $\begin{array}{r}20 \\
200 \\
20 \\
7 \\
4\end{array}$ & $\begin{array}{l}14619.96 \\
14610.28 \\
14495.63 \\
14470.26 \\
14370.36\end{array}$ & $\begin{array}{c}\text { 20. } 0 \\
10.3 \\
70.2 \\
--\end{array}$ & $\begin{array}{c}24_{2}^{\circ}-\mathrm{M}_{3} \\
8_{3}^{\circ}-e^{3} \mathrm{D}_{3} \\
24_{2}^{\circ}-e^{1} \mathrm{D}_{2}\end{array}$ & $\begin{array}{l}7977.35 \\
8093.86 \\
8204.47 \\
8224.78 \\
8227.55\end{array}$ & $\begin{array}{r}60 \\
7 \\
90 \\
400 \\
70\end{array}$ & $\begin{array}{l}12532.04 \\
12351.65 \\
12185.13 \\
12155.04 \\
12150.95\end{array}$ & $\begin{array}{l}32.1 \\
51.7 \\
85.1 \\
55.0 \\
50.9\end{array}$ & $\begin{aligned} & 30_{4}^{\circ}-\mathrm{N}_{4} \\
& 20_{4}^{\circ}-e^{5} \mathrm{~F}_{4} \\
& z^{5} \mathrm{~F}_{4}^{\circ}-e^{3} \mathrm{D}_{3} \\
& a^{1} \mathrm{G}_{4}-z^{3} \mathrm{~F}_{3}^{\circ} \\
& z^{5} \mathrm{D}_{2}^{\circ}-e^{3} \mathrm{D}_{2}\end{aligned}$ \\
\hline $\begin{array}{l}6957.51 \\
6975.70 \\
6989.83 \\
7012.03 \\
7030.08\end{array}$ & $\begin{array}{l}20 \\
15 \\
20 h \\
2 \\
10\end{array}$ & $\begin{array}{l}14368.99 \\
14331.53 \\
14302.56 \\
14257.27 \\
14220.67\end{array}$ & $\begin{array}{c}31.5 \\
-- \\
-- \\
--\end{array}$ & $b^{1} \mathrm{D}_{2}-z{ }^{3} \mathrm{D}_{3}^{\circ}$ & $\begin{array}{l}8259.03 \\
8301.83 \\
8428.47 \\
8456.36 \\
8619.91\end{array}$ & $\begin{array}{c}7 \\
20 \\
4 \\
3 h \\
4 h\end{array}$ & $\begin{array}{l}12104.63 \\
12042.23 \\
11861.29 \\
11822.17 \\
11597.85\end{array}$ & $\begin{array}{l}04.7 \\
42.2 \\
61.3 \\
22.2 \\
97.9\end{array}$ & $\begin{aligned} & 32_{3}^{\circ}-\mathrm{N}_{4} \\
& 29_{i}^{\circ}-\mathrm{G}_{1} \\
& 32_{3}^{\circ}-e^{1} \mathrm{D}_{2} \\
& 31_{2}^{\circ}-e^{3} \mathrm{D}_{1} \\
& z^{1} \mathrm{~F}_{3}^{\circ}-\mathrm{N}_{4}\end{aligned}$ \\
\hline $\begin{array}{l}7056.28 \\
7065.57 \\
7078.09 \\
7094.61 \\
7094.78\end{array}$ & $\begin{array}{r}5 \\
10 \\
8 \\
15 \\
100\end{array}$ & $\begin{array}{l}14167.87 \\
14149.24 \\
14124.21 \\
14091.32 \\
14090.98\end{array}$ & $\begin{array}{l}\text { 67. } 9 \\
49.3 \\
24.2 \\
-- \\
--\end{array}$ & $\begin{array}{c}27_{3}^{\circ}-\mathrm{M}_{3} \\
b^{1} \mathrm{D}_{2}-13_{2}^{\circ} \\
17_{3}^{\circ}-e^{5} \mathrm{~F}_{4}\end{array}$ & $\begin{array}{l}8722.99 \\
8762.47 \\
8804.61 \\
8981.87 \\
9004.06\end{array}$ & $\begin{array}{l}6 h \\
70 \\
2 \\
8 \\
7 h\end{array}$ & $\begin{array}{l}11460.81 \\
11409.17 \\
11354.57 \\
11130.48 \\
11104.29\end{array}$ & $\begin{array}{l}09.2 \\
54.5 \\
30.4 \\
--\end{array}$ & $\begin{array}{c}z^{3} \mathrm{D}_{3}-e{ }^{3} \mathrm{D}_{3} \\
z^{1} \mathrm{~F}_{3}^{\circ}-e{ }^{1} \mathrm{D}_{2} \\
b^{1} \mathrm{D}_{2}-8_{3}^{\circ}\end{array}$ \\
\hline $\begin{array}{l}7113.73 \\
7122.92 \\
7125.05 \\
7131.64 \\
7179.95\end{array}$ & $\begin{array}{r}400 \\
3 \\
5 \\
15 \\
4\end{array}$ & $\begin{array}{l}\text { 14053. } 45 \\
14035.32 \\
14031.12 \\
14018.16 \\
13923.84\end{array}$ & $\begin{array}{c}\text { 53. } 5 \\
-- \\
\text { 18. } 1 \\
23.9\end{array}$ & $\begin{array}{c}a^{3} \mathrm{P}_{1}-z^{3} \mathrm{P}_{2}^{\circ} \\
z^{2}{ }^{2} \mathrm{P}_{0}^{\circ}-e{ }^{\circ} \mathrm{D}^{3} \mathrm{D}_{1}\end{array}$ & $\begin{array}{l}9012.40 \\
9060.50 \\
9100.87 \\
9128.03 \\
9201.81\end{array}$ & $\begin{array}{l}3 h \\
1 \\
2 \\
2 h \\
20\end{array}$ & $\begin{array}{l}11092.78 \\
11033.89 \\
10984.94 \\
10952.26 \\
10864.44\end{array}$ & $\begin{array}{l}92.8 \\
85.0 \\
52.2 \\
64.5\end{array}$ & $\begin{array}{c}32_{3}^{\circ}-\mathrm{H}_{3} \\
32_{3}^{\circ}-e{ }^{5} \mathrm{~F}_{3} \\
32_{3}^{\circ}-\mathrm{Q}_{3} \\
z{ }^{3} \mathrm{P}_{1}^{\circ}-e{ }^{3} \mathrm{D}_{2}\end{array}$ \\
\hline $\begin{array}{l}7217.57 \\
7254.23 \\
7407.44 \\
7486.03 \\
7607.25\end{array}$ & $\begin{array}{c}200 \\
5 h \\
3 \\
80 \\
10\end{array}$ & $\begin{array}{l}\text { 13851. } 26 \\
13781.26 \\
13496.22 \\
13354.54 \\
13141.74\end{array}$ & $\begin{array}{c}51.3 \\
- \\
54.6 \\
41.8\end{array}$ & $\begin{array}{c}z{ }^{3} \mathrm{D}_{2}^{\circ}-e{ }^{3} \mathrm{D}_{2} \\
a^{1} \mathrm{G}_{4}-z{ }^{0} \mathrm{D}_{3}^{\circ} \\
\quad 27_{3}^{\circ}-e{ }_{5} \mathrm{~F}_{3}\end{array}$ & $\begin{array}{r}9291.30 \\
9340.16 \\
9460.64 \\
10005.95 \\
10539.57\end{array}$ & $\begin{array}{l}3 h \\
4 \\
3 h \\
7 h \\
2 h\end{array}$ & $\begin{array}{r}10759.80 \\
10703.52 \\
10567.21 \\
9991.31 \\
9485.45\end{array}$ & $\begin{array}{l}59.8 \\
03.5 \\
91.3\end{array}$ & 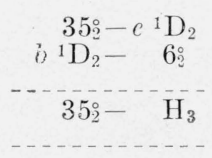 \\
\hline $\begin{array}{l}7614.83 \\
7618.17 \\
7626.20 \\
7637.65 \\
7641.27\end{array}$ & $\begin{array}{c}8 \\
30 h \\
4 h \\
7 h \\
2\end{array}$ & $\begin{array}{l}13128.65 \\
13122.90 \\
13109.08 \\
13089.43 \\
13083.23\end{array}$ & $\begin{array}{c}28.7 \\
09.0 \\
-- \\
-\end{array}$ & $\begin{array}{c}27_{3}^{\circ}-\mathrm{E}_{3} \\
27_{3}^{\circ}-O_{3}\end{array}$ & $\begin{array}{l}10546.35 \\
10685.35 \\
10705.51 \\
10757.78\end{array}$ & $\begin{array}{l}20 \\
2 \\
4 h \\
8\end{array}$ & $\begin{array}{l}9479.35 \\
9356.04 \\
9338.42 \\
9293.05\end{array}$ & $\begin{array}{c}79.4 \\
-- \\
-- \\
-\end{array}$ & $18 \mathrm{i}-c^{3} \mathrm{D}_{2}$ \\
\hline $\begin{array}{l}7644.11 \\
7738.60 \\
7749.76 \\
7761.63 \\
7780.53\end{array}$ & $\begin{array}{c}5 \\
3 h \\
40 \\
2 \\
70 h\end{array}$ & $\begin{array}{l}13078.37 \\
12918.68 \\
12900.07 \\
12880.35 \\
12849.06\end{array}$ & $\begin{array}{c}-- \\
00.0 \\
- \\
-\end{array}$ & $29_{1}^{\circ}-e{ }^{1} \mathrm{D}_{2}$ & & & & & \\
\hline
\end{tabular}

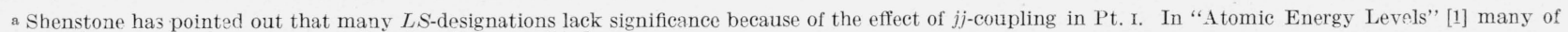
the earlier designation assignments are, therefore, being omitted.

\section{References}

[1] C. E. Moore, Atomic energy levels as derived from analyses of optical spectra, NBS Circular 467, III (in press).

[2] W. F. Meggers, BS Sci. Pap. 20, 31 (1925) S499.

[3] C. E. K. Mees, J. Opt. Soc. Am. 25, 80 (1935).

[4] R. J. Murphy, J. Research NBS 49, 371 (1952) RP2374.

[5] K. G. Kessler and W. F. Meggers (unpublished.).

[6] Transactions of the Joint Commission for Spectroscopy, J. Opt. Soc. Am. 43, 423 (1953).
[7] A. G. Shenstone, Phys. Rev. 36, 669 (1930).

[8] A. G. Shenstone, Proc. Roy. Soc. [A] 219, 419 (1953).

[9] A. C. Haussmann, Astrophys. J. 66, 333 (1927).

[10] W. F. Meggers and O. Laporte, Phys. Rev. 28, 642 (1926).

[11] H. Kayser, Handbuch der Spektroscopie 6, 304 (1912).

[12] F. Exner and E. Haschek, Die Spektren der Elemente

II, 204 (Franz Deuticke, Leipzig und Wien, 1911).

[13] J. J. Livingood, Phys. Rev. 34, 185 (1929).

Washington, June 6, 1954 